\title{
Ethics in Surgical Innovations from the Patient Perspective
}

\author{
Tony Eyers, Yordanka Krastev \\ Macquarie University, Sydney, Australia
}

\section{Summary}

Objective: Surgical innovation (SI) can place patients at risk. We sought to explore what clinical information is readily available to patients who have been offered innovative surgical procedures, using two examples drawn from our recent experience: one a surgical technique, and the other a prosthetic material. We wanted to determine from our review the extent to which information available on the Internet might augment the medical literature and help satisfy the ethical requirements for patients to be adequately informed before they proceed with innovative surgery. Methods: A scoping review of the medical literature was performed to look for studies addressing the review aims; targeted searches on Google, YouTube, and patient websites were carried out to find readily available patient information on two chosen innovative surgical procedures. We conducted a content analysis of the selected references to determine the availability, relevance, and the utility of the published information to a layperson.

Results: Medical database searches identified 614 records, 91 were screened and only six were relevant. The Internet searches returned thousands of results; however, we limited our screening to the first five pages of results for those sources. From both types of searches, 348 references were excluded because they did not meet the inclusion criteria and 51 were included in the analysis. The findings are presented in four themes: safety and feasibility of the technique, availability and accessibility to a layperson, relevance and utility to a layperson, and commercial information. Conclusion: The review has shown that lay people seeking to find out more about the two innovations would get very little useful information from Google, YouTube, or patient websites. Practitioners offering SI should provide sufficient information to allow their patients to make an autonomous decision about whether to proceed. For major Sl, we encourage innovators to develop a plain language statement that would be made available on the Internet to the mutual advantage of both innovators and patients.

\section{Keywords}

Ethics, surgical innovation, patient information, social media, internet

Yearb Med Inform 2020:169-75

http://dx.doi.org/10.1055/s-0040-1701994

\section{Introduction}

Surgical innovation (SI) is widely accepted as a "good" - something to be encouraged and facilitated in the public interest. But it involves a degree of experimentation and can put patients at risk. "Innovation" includes a spectrum extending from the original Idea (I), through Development (D), Exploration (E), Assessment (A) and Long-term (L) study, whose stages have been labeled with the acronym "IDEAL" by a collaboration of experts [1]. With IDEAL, lay people can appreciate the stages whereby new surgical procedures or devices are introduced into practice, and if a new procedure is offered, they can make an informed choice about undergoing that procedure. In addition, patients or patient advocacy groups can contribute to the discussions and recommendations of the IDEAL framework by sharing their perspectives, values, and experience [2]. While the later stages of SI are usually the subject of formal research, the early stages often occur without it, and we suggest that the ethical principles that apply to research involving human participants should apply equally to the early stages of SI.

The Australian National Statement on ethical conduct in human research defines the following ethical values and principles: research merit and integrity, justice, beneficence, and respect [3]. Using IDEAL, Rogers and her colleagues have aligned these principles to the SI spectrum, identifying four important areas for consideration [4, 5]: a) minimizing harm, b) autonomy and consent, c) justice issues, and d) managing conflicts of interest (See Table 1).

The principles indicate that care and planning are crucial in SI, including the gathering of information about the reasonably predictable risks and anticipated benefits of the proposal.
At present, any information statements are largely bespoke efforts but making information available on the internet presents opportunities to consolidate the efforts of many and facilitate their dissemination in a form accessible to the public.

\section{Objectives}

The purpose of the review was to explore what clinical information is readily available to patients who have been offered innovative surgical procedures and to determine the availability, relevance, and utility to a layperson, of information readily accessible on internet.

The authors have chosen two examples of innovative procedures representing the two main types of interventions being applied for by contemporary would-be innovators: innovative procedures and innovative devices. They were chosen because they were the subject of recent applications for oversight approval at the authors' institution, and thus were probably still at the "D" and/or "E" stages of the IDEAL spectrum. The chosen innovations are:

1) Transoral Endoscopic Thyroidectomy (TET) - an innovative surgical technique that accesses the thyroid gland from the mouth through the soft tissues of the neck, enabling it to be removed without leaving any disfiguring skin-scar on the neck.

2) Inguinal Hernia Repair with Mesh (IHRM) - a conventional surgical operation, but with a novel prosthetic material [Parietex${ }^{\text {TMProGrip }}{ }^{\mathrm{TM}}$ mesh] designed to be self-fixing in the surgical wound, and thus should not be needing to be sutured in place. This has the potential benefits of speeding the surgery and decreasing the risk of nerve, spermatic cord or vascular injuries. 
Table 1 Key ethical principles and applicability to surgical innovations. Source: Adapted from Rogers W, Hutchison K, McNair A [4].

\begin{tabular}{|l|l|}
\hline Ethical principles & Applicability to surgical innovations \\
\hline Minimizing harm & $\begin{array}{l}\text { Primarily concerned with harm to patients. In pre-IDEAL studies, harms to } \\
\text { animals may also be relevant. Reputational and psychological harms to health } \\
\text { professionals can follow unsuccessful innovation. } \\
\text { Autonomy and consent } \\
\text { Primarily concerned with ensuring rigorous processes of patient consent. This } \\
\text { includes informing patients of the innovative nature of the procedure and } \\
\text { providing transparency about the limits of existing knowledge and associated } \\
\text { risk of unexpected complications. } \\
\text { Concerned with fair distribution of the risks and benefits of surgical innovation. } \\
\text { Selection of patients for risky procedures must not target those who are } \\
\text { vulnerable. Access to successful innovations should be fair. } \\
\text { Concerned with impact of conflicts of interest on judgment. These may lead } \\
\text { to prioritizing surgeons' and device manufacturers' interests over those of } \\
\text { patients and also give rise to injustices. Conflicts of interest arise when surgical } \\
\text { innovation leads to financial and other rewards. }\end{array}$ \\
\hline
\end{tabular}

\section{Methods}

\section{Search Strategies}

We undertook a narrative review of the literature and conducted analysis following the methods outlined by Pare et al., [6] and Ferrari [7] to understand what information is available to the layperson about the selected surgical innovations and how it is presented.

We wanted to review reported outcomes of TET and IHRM. We also wanted to investigate reports of any patient decision aids or other patient decision-making processes related to these two innovations. We performed targeted searches on the electronic databases Medline, EMBASE, and Scopus to search for studies describing TET and new devices for IHRM. We used subject headings and keywords for the following concepts: innovative procedures, innovative devices, transoral endoscopic thyroidectomy, inguinal hernia repair, mesh, patient decision aids. We limited the search to English language articles published between January 2016 and September 2019. We hand searched reference lists and citing articles of relevant studies for additional papers. A second search of the Internet was conducted to obtain information on the two innovations that patients would most likely be able to access and understand. Internet searches included Google, You Tube, and patient social media sites: PatientsLikeMe [8] and Surgery squad [9].

\section{Screening and Quality Assessment}

The references derived from the databases and Internet searches were screened independently by both authors (YK and TE) according to the selection criteria. When it was not possible to determine whether a reference was relevant, it was included at this stage.

Internet searches on both TET and IHRM identified thousands of results. We limited our screening to the first five pages of results for those sources. References were excluded if they did not meet the inclusion criteria (See Table 2). Selected references for inclusion in the analysis were discussed and agreed upon by both authors.

Table 2 Inclusion and exclusion criteria used to identify patient information on Internet related to Transoral Endoscopic Thyroidectomy (TET) and Inguinal Hernia Repair with Mesh (IHRM).

\begin{tabular}{l|l|}
\hline Inclusion criteria & Exclusion criteria \\
\hline Available publications about & Patient materials not \\
TET and IHRM & related to TET or IHRM \\
Relevant patient materials & Very technical publi- \\
described in lay language & cations \\
Accessibility of information & Information not accessi- \\
to patients & ble to patients \\
Published in the English & Publication not in English \\
language & language \\
\hline
\end{tabular}

\section{Data Analysis}

We conducted a content analysis of the selected references in accordance with our objective to determine the availability, relevance, and utility of the published information to a layperson. We chose a narrative review format to report the results, as we aimed to identify and synthesize a broad range of literature available to patients as well as health professionals in the chosen clinical innovation areas and demonstrate the gaps in the knowledge, rather than seeking generalization of the outcomes [6]. The process of screening and the analysis of search results are depicted in the flow diagram displayed in Figure 1.

\section{Results}

The medical databases searches yielded 614 records, 91 were screened and only six were relevant. They were all focused on the safety and feasibility of the technique or the device. Altogether from both types of searches 327 references were excluded due to not meeting the inclusion criteria, and 51 were included in the analysis.

The findings of our analysis are presented in four themes: safety and feasibility of the technique, availability and accessibility to a layperson, relevance and utility to a layperson, and commercial information.

\section{Safety and Feasibility of the Technique}

Of the 40 screened titles and abstracts from the database searches related to TET, only two papers had a patient focus. The article by Bakkar et al., [10] concluded that patients who agree to undertake a novel surgical procedure should be properly counseled especially about unconventional procedure-related complications. A systematic review by Camenzuli et al., [11], including 785 patient cases, determined that the TET procedure is a safe and feasible technique and demonstrated acceptable complication rates and good outcomes. 


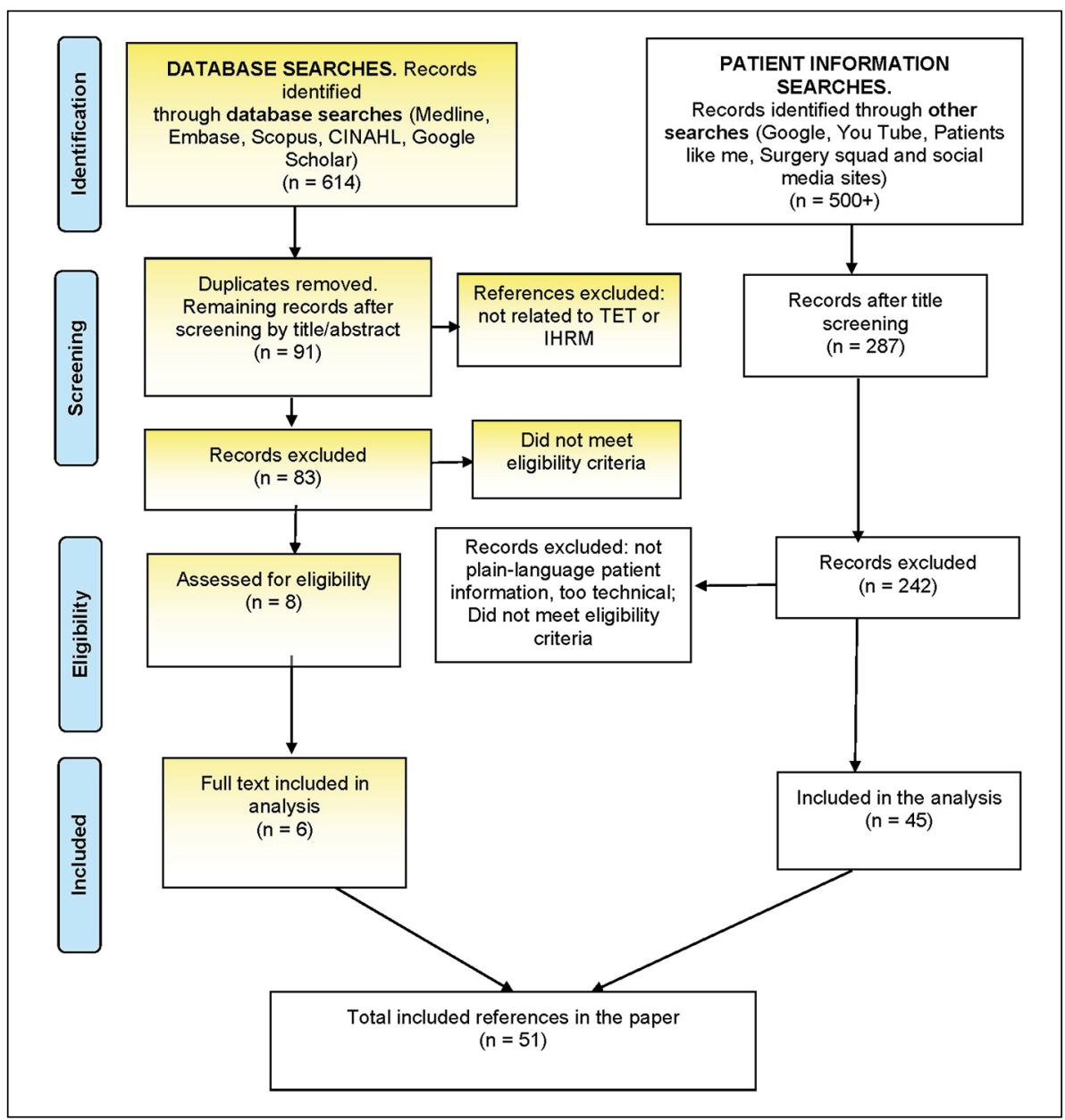

Fig. 1 Flow diagram of database and other sources searches.

From: Moher D, Liberati A, Tetzlaff J, Altman DG, The PRISMA Group (2009). Preferred Reporting Items for Systematic Reviews and Meta-Analyses: The PRISMA Statement. PLoS Med 6(6): e 1000097. doi:10.1371/journal.pmed 1000097. For more information, visit www.prisma-statement.org.

The Google search on TET led to a cluster of scientific articles and a video that concluded the technique was new, and under either evaluation or assessment (at "E" or "A" in IDEAL) [12-14]. It rapidly became clear that TET is a cluster of procedures and thus confusion could easily arise as to precisely which technique is being proposed, and what IDEAL stage the technique is at.

The following six different techniques we found could be grouped as "laparoscopic - or endoscopic thyroidectomy"

- TransOral Endoscopic Thyroid surgery Vestibular Approach (TOETVA),

- TransOral Endoscopic Thyroidectomy (TOET),
- TransOral Robotic Thyroidectomy(TORT),

- TransOral Robotic Surgery (TORS),

- TransOral and Submental Technique (TOaST), and

- Total Endoscopic Thyroidectomy via Areola approach (ETA), in contrast to "Conventional Open Thyroidectomy (COT)".

Database searches for IHRM identified 30 titles, only one of which passed screening. It was an observational study on laparoscopic ventral hernia repair using self-fixating mesh performed on 41 patients, which reported a decrease in the operative time, no complications, and a positive quality-of-life evaluation one month after the procedure [15].
The Google search for IHRM identified some scientific papers [16-18] that were beyond the comprehension of a layperson. There was also evidence of a litigious interest in the area of mesh repair [19]. Parietex ${ }^{\mathrm{TM} P r o-}$ Grip ${ }^{\mathrm{TM}}$ mesh was identified as an innovative product in the process of assessment (at "A" in IDEAL) [20]. A website called "Mesh Injured Australia" [21] cited a Cochrane review whose conclusion was: "Hernia repairs with and without mesh both proved effective in the treatment of hernias, although mesh repairs demonstrated fewer hernia relapses, a shorter operation time and faster return to normal activities. Non-mesh repairs are still widely used, often due to the low cost and limited availability of the mesh product itself' [22].

\section{Availability and Accessibility}

Google searches on TET and IHRM identified several specialized articles that were very technical. However, we found three patient discussion forums which were easily accessible to people who have undergone an inguinal hernia surgery [23-25] and the British Thyroid Association has a dedicated patient information page with leaflets on various thyroid-related conditions and links to professional organizations [26].

The Advances in Surgery channel [27] has video resources related to TET [28] and IHRM [29] but this site is very technical, and would probably be of limited value in informing laypersons on risks and benefits.

Google searches for patient forums for TET led to two chat threads published in support group forums $[30,31]$ and two informative consumer-oriented resources [32, 33]. From the YouTube searches we identified patient information on thyroidectomy in general, but not endoscopic thyroidectomy [34].

There were no patient-specific decision aids for TET or IHRM from the targeted database searches on patient education. We identified a Cochrane systematic review that analyzed a range of patient decision aids which can be applicable for the two selected examples. It concluded that patients exposed to decision aids would feel more knowledgeable, better informed, and clearer about their values and they would have more accurate risk perceptions [35]. 


\section{Relevance and Utility to a Layperson}

A study by Tamhankar et al., [36] found that a big proportion of patients undertaking common surgical procedures used the internet to inform themselves, and about one-third of them specifically searched for information about their operation. The information provided on the Internet can confuse and trouble patients.

Decision support resources that might be useful to a layperson considering both TET and IHRM were identified in the "Decision Aids Library Inventory" produced by the Ottawa Hospital [37]. Plain-language information on IHRM and patient resources were also available on the websites of organizations such as UCI (University of California Irvine) Health, American College of Surgeons, and US Food and Drug Administration [38-40].

For IHRM we found some informative videos on YouTube $[41,42]$ and two informative resources about inguinal hernia repair with or without mesh $[43,44]$. We also found a promotional/educational video, clearly sponsored by the manufacturer Coviden $^{\mathrm{TM}}$ [45] but nonetheless informative for a layperson.

The PatientsLikeMe website provides information about conventional thyroidectomy [46] and inguinal hernia repair [47]. One private patient Facebook support group "Hernia Mesh Australia" [48] offered membership to Australian men and women who have had hernias repaired and are suffering resultant pain. The Surgery Squad platform [9] provides surgery games and some patient education, but when accessed, none of the 34 available posts were related to thyroidectomy or hernia repair and as well changed to "Commercial Information".

\section{Commercial and Litigation Information}

Internet searches for IHRM led to two pages of the mesh manufacturer's website [49, 50] that were inaccessible to a layperson. A Google search on "should I have mesh hernia surgery?" led to a significant number of sites reflecting a widespread concern about its use $[51,52]$. We found evidence of litigation [53] and a BBC documentary [54] on "Parietex-
Mesh that appears to have been posted by BioHernia, an organization promoting and offering hernia repair using established techniques which do not use mesh. We felt that the evidence of controversy would lead a reasonable enquirer to ask patients whether they should have their hernia repaired with mesh. Health Issues Centre, a consumer advocacy organization offered participation in a survey, "to identify other patterns of malfunction in medical devices and implants" [55].

\section{Discussion}

\section{Ethical Considerations and Implications for Practice}

In its early stages, SI has little or no evidence-base, and (as in Phase 1 clinical trials) it is important that expected outcomes are likely to outweigh the known (or reasonably predictable) risks, that the outcomes will be assessed and recorded adequately, that the patient's proper informed consent has been obtained, and that the voluntary nature of their participation is confirmed.

SI has some special aspects which play into these ethical considerations, -quantum of change, diffusion of innovation, and advocacy issues.

\section{Quantum of Change}

Much SI is minor and represent little more than an incremental change. These are very common in surgical practice, and there is yet no clear understanding of what constitutes a treatment "variation" as opposed to an "innovation" of enough magnitude to warrant a deliberate plan and formal oversight [56]. The more major the proposed SI is, the more important becomes full adherence to the principles.

\section{Diffusion of Innovation}

"Diffusion" is "a social process through which cultural knowledge, practices, and materials spread in social systems" [57]. In 1962, Everett Rogers [58] applied it to innovative farming techniques and concluded that "in a social system, a decision to adopt an innovation depends heavily on decisions made by other members of the system". He argued people were influenced more by their peers than by the evidence. He identified those involved as "innovators", "early adopters", "the early majority", "the late majority", and "laggards", and dispersed them in his now well-known bell-curve (See Figure 2) according to their propensity to either adopt or resist change. Rogers' work achieved wide acceptance and is taught and

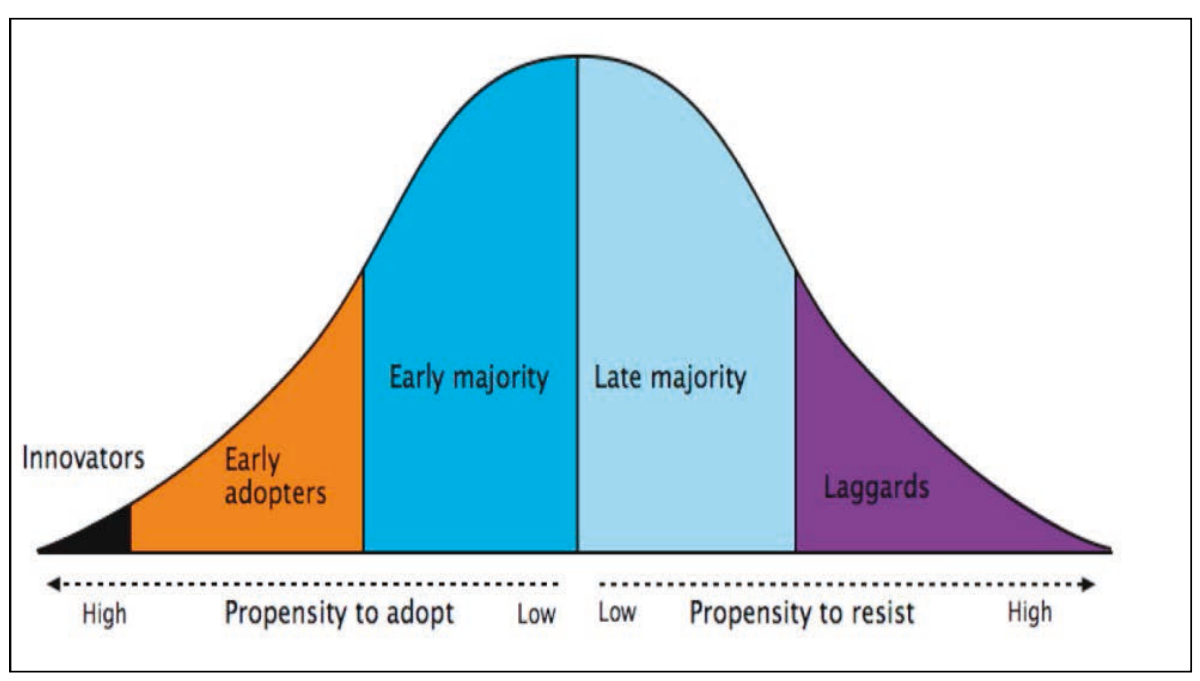

Fig. 2 Different adopter categories.

Source: http://sphweb.bumc.bu.edu/ott//MPH-Modules/SB/BehavioralChangeTheories/BehavioralChangeTheories4.html 
practiced in contemporary marketing [59]. It is often applied to SI, making it important that both innovators and their patients appreciate that peer pressure and marketing may be influencing the SI.

\section{Advocacy Issues}

Most SI take place without sponsorship by independent third parties. Practitioners offering the SI may also be its sponsors, but more often they are advocates of the procedure and stand to benefit professionally from its success. If the innovator is the inventor of the SI (and thus might have Intellectual Property claims), is providing any support (whether financial or support in kind), or stands to benefit from its success, there are potential conflicts of interest. Ethically, these must be declared and managed appropriately.

\section{Implications for Practice}

Each of the three elements discussed above can affect the informed consent process adversely, and in SI the consent process warrants particularly close attention. Patients must understand what is going to be done differently, what are the likely benefits and potential risks, and need to be assured of the voluntary nature of their participation.

Innovators have the option of giving their patients procedure-specific handouts as part of the informed consent process. When decision aids are offered to patients, it improves their knowledge and helps achieve shared treatment decisions [60]. Procedure-specific handouts are emphasized in the "Guide to good practice for surgical innovation, new techniques, and technologies" published by The Royal College of Surgeons of England [61]. The guide offers a framework and a decision tree for surgeons, their teams, and hospital clinical managers, on what to consider when developing a new surgical technique or innovation and the ethical, regulatory, patient consent, and cost requirements. SI is an area where patient-directed information would be extremely useful.

However, as Alsaffar et al., noted, a pamphlet alone might not be enough and should be supplemented by additional patient education using emerging technologies [62]. This might be challenging for many would-be innovators. According to Hoffman et al., "emerging evidence suggests potential benefits to delivering patient decision aids on the Internet. However, additional research is needed to identify best practices and quality metrics for Internet-based development, evaluation, and dissemination, particularly in the areas of interactivity, multimedia components, socially generated information, and implementation strategies" [63].

\section{Conclusions}

This review has confirmed that lay people seeking to find out about the two selected examples of SI currently in their early stages would get very little useful information from Google, YouTube or social media sites. However, due to the focus on just two clinical examples in our narrative review, the results are not generalizable to other surgical innovations.

Lay people would be able to connect with patients who have issues with various aspects of their care and with individuals in recovery from surgery which appears to have gone well enough. They would also encounter a plethora of promotional material and various other options which may not be at all relevant to their situation.

It is an ethical requirement of practitioners offering SI to provide sufficient information to allow their patients to make an autonomous decision to proceed. We believe a good description of the procedure, including the potential risks and benefits, the experience of the clinical team, and any available alternatives, can be very beneficial for the patient and for the successful introduction of the innovation.

There is clearly a place for the use of electronic media to disseminate patient decision aids and make them more accessible, but this is still in its infancy [63]. At present, persistence would be required to find reputable information, including decision aids, but even that seems unlikely to be able to directly inform patients regarding the risks and benefits of the innovation.
For major or significant innovations, we encourage practitioners to develop a plain-language statement and make it available on the Internet to the mutual advantage of both innovator and patient.

Our sample was limited, but for these two specific SIs the Internet was not helpful. But we did encounter the Ottawa Hospital site [37] which provides an example of a useful resource. We advocate the development of sites which focus on innovative treatments, enabling practitioners trying to develop decision aids for SI to collaborate and promulgate patient-directed information.

\section{Strengths and Limitations}

The authors are members of an institution with an established interest in the ethics of surgical innovation. Both are members of an Institutional Human Research Ethics Committee, and of a hospital committee which assesses and provides approval for innovative surgical procedures.

Due to time restrictions, the authors selected two recently proposed innovations representing the two broad categories of SI and performed a descriptive analysis of the findings, rather than using an online health information scale to evaluate the SIs.

\section{Acknowledgements}

We would like to thank Ms. Mary Simons, Clinical Librarian, Faculty of Medicine, Health and Human Sciences, Macquarie University, who performed the searches of the medical databases and advised on the methodology section of the paper.

\section{References}

1. The IDEAL Collaboration. Idea, Development, Exploration, Assessment, Long-term Follow-up, Improving the Quality of Research in Surgery. [Internet] [cited 202024 April]. Available from: $\mathrm{http}: / /$ www.ideal-collaboration.net/

2. The IDEAL Collaboration. What IDEAL means for you. The public. [Internet] [cited 202024 April]. Available from: http://www.ideal-collaboration. net/what-ideal-means-for-you/

3. National Health and Medical Research Council. National Statement on Ethical Conduct in Human Research 2007 (updated 2018); 2018.

4. Rogers W, Hutchison K, McNair A. Ethical Issues Across the IDEAL Stages of Surgical Innovation. 
Ann Surg 2019;269:229-33.

5. Johnson J, Rogers W, Lotz M, Townley C, Myerson D, Tomossy G. Innovative Surgery: the ethical challenges. J Med Ethics 2012;38(12).

6. Paré G, Kitsiou S. Chapter 9. Methods for Literature Reviews. In: Lau F, Kuziemsky C, editors. Handbook of eHealth Evaluation: An Evidence-based Approach [Internet] Victoria (BC): University of Victoria; $2017 \mathrm{Feb} 27$. [Available from: https://www.ncbi.nlm.nih.gov/books/ NBK481583/

7. Ferrari R. Writing narrative style literature reviews. Medical Writing 2015;4(24):230-5.

8. PatientsLikeMe. 2019 [Cited 2019 September 26]. Available from: https://www.patientslikeme.com/ conditions/thyroid-ca

9. Surgery Squad. Surgery games \& patient education. \{Internet] 2018 [Cited 2019 October 31]. Available from: https://www.surgerysquad.com/ category/surgery-games/page/3/

10. Bakkar S, Al Hyari M, Naghawi M, Corsini C, Miccoli P. Transoral thyroidectomy: a viable surgical option with unprecedented complications-a case series. J Endocrinol Invest 2018;41(7):809-13.

11. Camenzuli C, Schembri Wismayer P, Calleja Agius J. Transoral Endoscopic Thyroidectomy: A Systematic Review of the Practice So Far. JSLS 2018;22(3):e2018.00026.

12. Pai VM, Muthukumar P, Prathap A, Leo J, A R. Transoral endoscopic thyroidectomy: A case report. Int J Surg Case Rep 2015;12:99-101.

13. Kadem SG, Habash SM, Jasim AH. Transoral Endoscopic Thyroidectomy via Vestibular Approach: A series of the first ten cases in Iraq. Sultan Qaboos Univ Med J 2019;19(1):e68-e72.

14. Wallace K. Transoral Endoscopic Thyroidectomy Vestibular Approach (TOETVA). [Internet] 2019 [cited 201928 October ]. Available from: https://medtube.net/general-surgery/medical-videos/25302-transoral-endoscopic-thyroidectomy-vestibular-approach-toetva

15. Muysoms F, Van Cleven S, Pletinckx P, Ballecer C, Ramaswamy A. Robotic transabdominal retromuscular umbilical prosthetic hernia repair (TARUP): observational study on the operative time during the learning curve. Hernia 2018;22(6):1101-11

16. Batabyal P, Haddad RL, Samra JS, Wickins S, Sweeney E, Hugh TJ. Inguinal hernia repair with Parietex ProGrip mesh causes minimal discomfort and allows early return to normal activities. Am JSurg 2016;211(1):24-30.

17. Yilmaz A, Yener O, Kaynak B, Yiğitbaşi R, Demir M, Burcu B, et al. Self-gripping Covidien ${ }^{\mathrm{TM}}$ ProGrip $^{\mathrm{TM}}$ mesh versus polypropylene mesh in open inguinal hernia repair: multicenter short term results. Prague Med Rep 2013;114(4):231-8.

18. Foo DCC. Comparison of Different Meshes in Laparoscopic Hernia Repair. [Internet]: Clinical Trials.gov, ; 2016 [cited 201931 October]. Available from: https://clinicaltrials.gov/ct2/show/ NCT02712827

19. Hollis Law. Parietex ProGrip Lawsuit: Who is the FDA Protecting? [Internet] [Cited 2019 October 31]. Available from: https://hollislawfirm.com/ case/hernia-mesh-lawsuit/parietex-progrip/

20. Barkun J, Aronson J, Feldman L, Maddern G,
Strasberg S. Surgical Innovation and Evaluation 1. Evaluation and Stages of Surgical Innovation. The Lancet 2009;374:1089-96.

21. Mesh Injured Australia. Comparing surgical groin hernia repair performed with or without mesh $\{$ Internet] [Cited 2019 October 25]. Available from: https://meshinjuredaustralia.org.au/reviews-research/

22. Lockhart K, Dunn D, Teo S, Ng JY, Dhillon M, $\mathrm{Teo} \mathrm{E}$, et al. Mesh versus non-mesh for inguinal and femoral hernia repair. Cochrane Database of Systematic Reviews. 2018(9).

23. Whirlpool. Inguinal hernia surgery [Internet] 20172019 [Cietd 2019 October 31]. Available from: https://forums.whirlpool.net.au/archive/2669630

24. Patientslikeme. Inguinal hernia [Internet] 2019 [Cited 2019 October 31]. Available from: https:// www.patientslikeme.com/conditions/inguinal-hernia

25. SlowtwitchTriathalon Forum. Inguinal hernia and recovery [Internet] [Cited 2019 October 31]. Available from: https://forum.slowtwitch.com/ forum/Slowtwitch_Forums_C1/Triathlon_Forum_ F1/_inguinal_hernia_and_recovery_P4538579/

26. British Thyroid Foundation. Your guide to Thyroid surgery. [Internet] 2018 [cited 201914 October]. Available from: https://www.btf-thyroid.org/thyroid-surgery

27. Advances in Surgery Channel. [Internet]. Barcelona2019 [cited 201914 October]. Available from: https://aischannel.com/

28. Lillo J.J., Martín-Pérez B. 5 things to know about transoral endoscopic thyroidectomy vestibular approach (TOETVA). [Internet] 2018 [cited 2019 14 October]. Available from: https://aischannel. $\mathrm{com} /$ society/5-things-know-transoral-endoscopic-thyroidectomy-vestibular-approach-toetva/.

29. Termes R. Mesh materials and types for hernia repair. [Internet] 2019 [updated 5 September 2019; cited 201914 October]. Video]. Available from: https://aischannel.com/society/mesh-materials-types-hernia-repair/

30. Cancer research UK. Thyroid surgery [Internet] 2019 [Cited 2019 October 14]. Available from: https://www.cancerresearchuk.org/about-cancer/ cancer-chat/thread/thyroid-surgery

31. Inspire. Thyroid diseases [Interenet] 2019 [Cited 2019 October 14]. Available from: https://www. inspire.com/groups/thyroid-diseases/

32. Hull N. Hyperthyroidism [Internet]: British Thyriod Foundation; [Cited 2019 October 14]. Available from: https://www.btf-thyroid.org/natasha-hull-hyperthyroidism

33. Thyriod Cancer Canada. Surgery or Thyroidectomy [Internet] [Cited 2019 October 14]. Available from: https://www.thyroidcancercanada.org/en/ treatments/surgery.

34. Mayo Clinic. Thyroidectomy [Internet] updated 2019 [Cited 2019 October 14]. Available from: URL: https://www.mayoclinic.org/tests-procedures/thyroidectomy/about/pac-20385195

35. Stacey D, Légaré F, Lewis K, Barry MJ, Bennett $\mathrm{CL}$, Eden KB, et al. Decision aids for people facing health treatment or screening decisions. Cochrane Database of Systematic Reviews. 2017(4):Art. No.: CD001431.

36. Tamhankar AP, Mazari FA, Everitt NJ, Ravi K. Use of the internet by patients undergoing elective hernia repair or cholecystectomy. Ann R Coll Surg Engl 2009;91(6):460-3.

37. The Ottawa Hospital. "Decision Aids Library Inventory" [Internet] [Cited 2019 October 23]. Available from: https://decisionaid.ohri.ca/cochinvent.php.

38. UCI Health. Is surgical mesh safe for my hernia surgery? [Internet] 2018 [Cited 2019 October 25]. Available from: https://www.ucihealth.org/ blog/2018/06/mesh-hernia-repair

39. American College of Surgeons. Groin hernia repair [Internet] [Available from: https://www. facs.org/ /media/files/education/patient $\% 20 \mathrm{ed} /$ groin_hernia.ashx

40. US Food and Drug Administration. Hernia Surgical Mesh Implants [Internet] 2018 [Cited 2019 October 25]. Available from: https://www.fda. gov/medical-devices/implants-and-prosthetics/ hernia-surgical-mesh-implants

41. Cleveland Clinic Abu Dhabi. Inguinal Hernia Repair [Internet] 2017 [Cited 2019 October 31]. Available from: https://www.youtube.com/ watch? $\mathrm{v}=\mathrm{hM}$-WA6WOw1Y

42. Johns Hopkins Medicine. Repairing a Hernia with Surgery [Internet] 2015 [Cited 2019 October 31]. Available from: https://www.youtube.com/ watch? $v=p L w 3 A j Z x 3 N Q$

43. California Hernia Specialists: Specialty Care for Hernia Repair. The Truth About Hernia Mesh: Why is Using Mesh Important? [Internet] 2015 [Cited 2019 October 31]. Available from: https://www. youtube.com $/$ watch? $\mathrm{v}=\mathrm{vKEXeXRJs-w}$

44. BioHernia. Inguinal Hernia Repair - With or without mesh? [Internet] 2017 [Cited 2019 October 31]. Available from: https://www.youtube.com/ watch? $\mathrm{v}=8 \_$pNhi80nJM.

45. Parietex ProGrip ${ }^{\mathrm{TM}}$. Anatomical Self Fixating Mesh. [Internet] 2014 [cited 201931 October ]. Available from: https://www.youtube.com/ watch? $\mathrm{v}=\mathrm{GX} 6$ YAld Y soY

46. Patientslikeme. What is Thyroidectomy? [Internet] [Cited 2019 September 26]. Available from: https:// www.patientslikeme.com/treatment/thyroidectomy

47. Patientslikeme. Inguinal hernia [Internet] [Cited 2019 September 26]. Available from: https://www. patientslikeme.com/conditions/inguinal-hernia

48. Hernia Mesh Australia (Support group). 2019 [Available from: https://www.facebook.com/ groups/310037816438647/

49. Medtronic. ProGrip ${ }^{\mathrm{TM}}$ Laparoscopic Self-Fixating Mesh [Internet] [Cited 2019 Otober 31]. Available from: https://www.medtronic.com/covidien/ en-au/products/hernia-repair/progrip-laparoscopic-self-fixating-mesh.html

50. Medtronic. MESH PRODUCTS [Internet] 2019 [Cited 2019 October 31]. Available from: https:// www.medtronic.com/covidien/en-au/products/ hernia-repair/mesh-products.html

51. Wakatama G. Concern grows over hernia mesh as more patients share post-surgery horror stories. [Internet]: ABC Newcastle; 2018 [cited 201925 October]. Available from: https://www.abc.net.au/ news/2018-09-14/hernia-mesh-patients-speak-outabout-horrendous-outcomes/10242004

52. Landro L. Hernia Repair: Secrets Patients Should Know. [Internet]: Meshnewsdesk; 2012 [cited 201925 October]. Available from: https://www. meshmedicaldevicenewsdesk.com/hernia-repair- 
secrets-patients-should-know/

53. Hollis Law Firm. Parietex ProGrip Hernia Mesh Unboxing \& Lawsuit Explanation \{Internet] 2017 [Cited 2019 October 31]. Available from: https://www.youtube.com/watch?v=BqjxPskAcMs\&t $=358 \mathrm{~s}$

54. BBC. BBC documentary about hernia meshes [Internet] 2017 [Cited 2019 October 31]. Available from: https://www.youtube.com/ watch?v=UsG-Kftd7ZM

55. Health Issues Centre. Medical Devices: You may not be alone in your medical device experience. [Internet] [Cited 2019 October 25]. Available from: https://www.surveymonkey.com/r/SKRYTGN

56. Hutchison K, Rogers W, Eyers T, Lotz M. Getting Clearer About Surgical Innovation: a new definition and a new tool to support safer practice. Ann Surg 2015;262(6):949-54.

57. Jussi K. Gabriel Tarde as a Founding Father of Innovation Diffusion Research. Acta Sociologica
1996;39(4):431-42.

58. Boston University School Public Health. Diffusion of Innovation Theory. http://sphweb.bumc.bu.edu/ otlt/MPH-Modules/SB/BehavioralChangeTheories/BehavioralChangeTheories4.html 2019

59. Peres R, Muller E, Mahajan V. Innovation diffusion and new product growth models: A critical review and research directions. International Journal of Research in Marketing 2010;27:91-106.

60. Knops AM, Legemate D, Goossens A, Bossuyt P, Ubbink D. Decision aids for patients facing a surgical treatment decision: a systematic review and meta-analysis. Ann Surg 2013;257(5):860-6.

61. Royal College of Surgeons of England. Surgical innovation, new techniques and technologies, A Guide to Good Practice. [Internet] 2019 [cited 201931 October]. Available from: https:// www.rcseng.ac.uk/standards-and-research/standards-and-guidance/good-practice-guides/surgical-innovation/
62. Alsaffar H, Wilson L, Kamdar DP, Sultanov F, Enepekides D, Higgins KM. Informed consent: do information pamphlets improve post-operative risk-recall in patients undergoing total thyroidectomy: prospective randomized control study. J Otolaryngol Head Neck Surg 2016;45:14.

63. Hoffman AS, Volk RJ, Saarimaki A, Stirling C, Li LC, Härter M, et al. Delivering patient decision aids on the Internet: definitions, theories, current evidence, and emerging research areas. BMC Med Inform Decis Mak 2013;13 Suppl(Suppl 2):S13.

\section{Correspondence to:}

Dr. Yordanka Krastev

MQ Health and Faculty of Medicine Health and Human Sciences Macquarie University

Level 3, 75 Talavera Road, Macquarie Park NSW 2113

Australia

E-mail: yordanka.krastev@mq.edu.au 